\title{
Experiencia docente en la formación inicial del profesorado: contribuciones a la
}

\section{enseñanza de la Educación Ambiental}

Teaching experience in initial teacher training: contributions to the teaching of Environmental

Education

Experiência docente na formação inicial de professores: contribuições para o ensino da Educação

Ambiental

Received: 09/02/2021 | Reviewed: 09/09/2021 | Accept: 09/11/2021 | Published: 09/13/2021

Karine Rosália Felix Praça Gomes ORCID: https://orcid.org/0000-0003-3491-1606 Universidade de Pernambuco, Brasil E-mail: karine.rosalia@upe.br

Stella Maria do Nascimento ORCID: https://orcid.org/0000-0003-0967-7344 Universidade de Pernambuco, Brasil E-mail: stella.nascimento@upe.br Emanuel Ferreira Leite

ORCID: https://orcid.org/0000-0002-3086-2002 Universidade de Pernambuco, Brasil E-mail: emanueleite@hotmail.com

Ana de Lourdes Torralbas Blazquez ORCID: https://orcid.org/0000-0002-7571-5954 Universidad de Holguín, Cuba E-mail: anatbcuba@gmail.com

María de Los Ángeles Cervantes Rosas ORCID: https://orcid.org/0000-0003-3338-4816 Universidad Autónoma de Occidente, México E-mail: maria.cervantes@uadeo.mx

\begin{abstract}
Resumen
Este estudio investigó la formación de los estudiantes de pregrado en Ciencias Biológicas relacionadas con el tema transversal del medio ambiente, experiencia vivida durante el desarrollo de la disciplina Práctica Docente $\mathrm{V}$ en la Facultad de Ciencia y Tecnología Profesor Dirson Maciel de Barros. El objetivo fue analizar los aportes formativos para los estudiantes de pregrado de biología de la experiencia docente en Educación Básica con la enseñanza de la Educación Ambiental. En la disciplina, se destacó el contenido programático relativo a los Temas Transversales, que se desarrollaron en forma de proyectos de extensión en escuelas públicas del municipio de Goiana-PE, promoviendo así la asociación entre Universidad y Escuela como forma de proporcionar a los estudiantes la oportunidad inicial de interacción con su futuro campo de actuación profesional. La investigación cualitativa se caracterizó en cuanto a los fines como descriptiva y los medios como estudio de campo. A partir de las experiencias en el aula, los alumnos realizaron proyectos con el tema de la Educación Ambiental y los aplicaron en las escuelas públicas estatales del municipio de Goiana-PE. Como resultado, los proyectos elaborados por los alumnos en las escuelas contenían propuestas metodológicas y didácticas para la enseñanza que pueden ser desarrolladas posteriormente por los profesores. Se concluye que en la experiencia docente el profesor construye su conocimiento a partir de la relación con los alumnos y la realidad escolar. Además, podrían contribuir al proceso de enseñanza-aprendizaje de los alumnos de educación básica y, principalmente, a su propio aprendizaje.

Palabras clave: Tema transversal; Formación del profesorado; Enseñanza de las Ciencias Naturales.
\end{abstract}

\begin{abstract}
This study investigated the training of undergraduate students in Biological Sciences related to the cross-cutting theme of the environment, an experience experienced during the development of the discipline Teaching Practice $\mathrm{V}$ in the Faculty of Science and Technology Professor Dirson Maciel de Barros. The objective was to analyze the formative contributions for the biology undergraduate students of the teaching experience in Basic Education with the teaching of Environmental Education. The course emphasized the programmatic content related to Cross-Cutting Themes, which were developed in the form of extension projects in public schools in the municipality of Goiana-PE, thus promoting the partnership between University and School as a way to provide undergraduates the initial opportunity to interact with their future field of professional activity. The research, qualitative in nature, was characterized in its ends as
\end{abstract}


descriptive and in its means as a field study. From the experiences in the classroom, the students made projects with the theme of Environmental Education and applied them in state public schools in the city of Goiana-PE. As a result, the projects developed by the students in the schools contained methodological and didactic teaching proposals that can later be developed by teachers. It can be concluded that, during the teaching experience, the teacher constructs his or her knowledge based on the relationship with the students and the school reality. In addition, they were able to contribute to the teaching-learning process of the basic education students and, mainly, to their own learning.

Keywords: Cross-cutting theme; Teacher education; Natural Science teaching.

\section{Resumo}

Este estudo investigou a formação de acadêmicos do curso de Licenciatura em Ciências Biológicas relacionada ao tema transversal do meio ambiente, experiência vivenciada durante o desenvolvimento da disciplina Prática de Ensino V na Faculdade de Ciências e Tecnologia Professor Dirson Maciel de Barros. O objetivo foi analisar as contribuições formativas para os acadêmicos do curso de licenciatura em biologia da experiência docente na Educação Básica com o ensino da Educação Ambiental. Na disciplina, enfatizou-se o conteúdo programático referente aos Temas Transversais, os quais foram desenvolvidos sob a forma de projetos de extensão em escolas públicas do município de Goiana-PE, promovendo, assim, a parceria entre Universidade e Escola como forma de propiciar aos licenciandos a oportunidade inicial de interação com seu futuro campo de atuação profissional. A pesquisa de cunho qualitativo, caracterizou-se quanto aos fins como descritiva e aos meios como um estudo de campo. A partir das vivências em sala de aula, os acadêmicos confeccionaram projetos com a temática da Educação Ambiental e aplicaram em escolas públicas estaduais no município de Goiana-PE. Como resultado, os projetos desenvolvidos pelos acadêmicos nas escolas continham propostas metodológicas e didáticas de ensino que posteriormente poderão ser desenvolvidas pelos docentes. Concluise, que na experiência docente o professor constrói seus saberes a partir da relação com estudantes e com a realidade escolar. Além disso, puderam contribuir para o processo de ensino-aprendizagem dos alunos da educação básica e, principalmente, para o seu próprio aprendizado.

Palavras-chave: Tema transversal; Formação de professores; Ensino em Ciências Naturais.

\section{Introducción}

La formación del profesorado en el contexto brasileño es un tema ampliamente debatido. Frente a este contexto, la estructuración de los planes de estudio para la formación del profesorado supone un reto para las instituciones de formación. Los planes de estudio tienen que estar en consonancia con las directrices para la formación de profesores de Educación Básica, como la Base Curricular Nacional Común para la Educación Primaria (Brasil, 2019), así como para la Educación Secundaria (Brasil, 2018) y también tener en cuenta la complejidad del contexto social en el que vivimos. La formación debe contemplar las preocupaciones por los derechos humanos, las tecnologías digitales de la información y la comunicación, la conciencia social y medioambiental, la pluralidad cultural, los temas transversales, entre otros indispensables para la formación de la ciudadanía y para vivir en la sociedad actual.

Cuando se piensa en la formación del profesorado, se reconoce que la institución educativa es una de las instancias que contribuye a la construcción de su conocimiento; pero no la única. Muchas concepciones sobre lo que es "ser profesor", traídas por los académicos, arrastran estigmas de la época en que fueron estudiantes de Educación Básica, necesitando ser problematizadas en relación a las teorías pedagógicas. Entre las diversas propuestas relativas a la formación del profesorado, tanto en las recientes directrices de formación como entre los teóricos, un aspecto ha sido común: la importancia de la dimensión de la experiencia docente en la formación del profesorado.

En este sentido, esta investigación se propone avanzar en la discusión del tema de la formación docente, delimitándolo a partir de una investigación realizada con estudiantes de pregrado de Ciencias Biológicas y, específicamente, en lo que respecta a la formación relacionada con el tema "Medio Ambiente". La preocupación con la enseñanza de este tema transversal ganó visibilidad en la educación brasileña a partir de los Parámetros Curriculares Nacionales (PCNs) (Brasil, 1997) y fue reforzada por la política nacional de Educación Ambiental (Brasil, 1999).

La cuestión medioambiental se ha debatido con mayor frecuencia, en tanto que se ha demostrado que la interferencia individual en los sistemas naturales puede comprometer tanto la satisfacción de la población mundial actual como la existencia 
de las generaciones futuras (Gomes et al, 2020). En este sentido, afirma que pensar en la cuestión medioambiental es, al mismo tiempo, repensar la forma de afrontar la vida en la Tierra, como comunidades, y sus retos y perspectivas de futuro.

Según dos Santos, Faria y Neiman (2020), la educación ambiental (EA) plantea, entre los diferentes actores sociales, la discusión sobre quién es responsable del cuidado del medio ambiente. En este sentido, muchas escuelas realizan actividades en este ámbito e intentan construir, junto con sus alumnos, conceptos sobre las responsabilidades hacia el entorno socioambiental, que a veces se reflejan en acciones positivas para la naturaleza. Ya que la función básica del profesor es proporcionar a los alumnos el aprendizaje y esto es una responsabilidad difícil (Gomes et al, 2020). En este sentido, los profesores son modelos de conducta y tienen un papel importante en el entorno social, interfiriendo directamente en el comportamiento de sus alumnos.

En cuanto a las corrientes teóricas de la Educación Ambiental, se entiende, así como Sauvé (2017) llama "corrientes" a las distintas posibilidades de pisar la Educación Ambiental, siendo ellas: Humanista, Conservacionista, Sistémica, Problematizadora, Naturalista, Científica, Moral, Biorregionalista, Sostenible, Crítica, Etnográfica, Feminista, entre otras propuestas.

También según Gomes et al (2020) la educación, en términos de ciudadanía, actúa como un factor crucial en la formación de una ciudadanía participativa. En este sentido, siendo un segmento de la educación, la Educación Ambiental es un locus que, a través de las interacciones educativas, actúa crucialmente en la formación de sujetos participativos, activos y capaces de asumir responsabilidades en términos de derechos y deberes.

En refuerzo teórico destaca la comprensión de Layrargues (2018), cuando identifica y organiza las características, potencialidades y trayectorias de tres macrotendencias político-pedagógicas de la Educación Ambiental: conservacionista, pragmática y crítica. En cada macrotendencia de la Educación Ambiental se expresa una concepción sobre el medio ambiente, según Reigota (2017), la forma de pensar y percibir el medio ambiente tiene influencia directa en los recorridos pedagógicos que se realizan en el aula. Esto trae a colación la relevancia del desarrollo de actividades de investigación que indaguen las concepciones de los docentes en ejercicio, es decir, cómo estos profesionales entienden el medio ambiente, la Educación Ambiental y fundamentan sus prácticas de enseñanza frente a las problemáticas socioambientales.

El planteamiento del tema del Medio Ambiente como tema tratado transversalmente en el aula orienta al profesor a los debates sobre los aspectos que pueden influir eficazmente en el método de sensibilización ambiental del alumno en el espacio escolar. Debatir sobre temas medioambientales requiere un sentido crítico, así como una visión local/global, conectando los conceptos científicos con la realidad de los alumnos, facilitando la comprensión del papel del hombre en la naturaleza.

En este entendimiento, la Educación Ambiental es señalada por los Parámetros Curriculares Nacionales - PCNs, como un tema transversal, debido a su viabilidad de abordaje en diversas áreas del conocimiento, que puede plantear cuestiones en relación con otros campos de estudio, y comunes a la sociedad. Sin embargo, es notable en la enseñanza de las ciencias, porque las Ciencias Naturales asocian fácilmente el tema de discusión con las demandas sociales y rutinarias del estudiante. Así, presenta contenidos con el objetivo de contribuir a una conciencia crítica de los alumnos en relación con las cuestiones globales, que implican el cuidado del medio ambiente y de las especies, así como los valores, las responsabilidades, la solidaridad, las nociones de ciudadanía y la salud.

Así, este estudio tuvo como objetivo analizar los aportes formativos para los estudiantes de pregrado en ciencias biológicas de la experiencia docente en Educación Básica con la enseñanza de la Educación Ambiental. Desde esta perspectiva, se piensa en la educación ambiental como una forma de transformar la sociedad, ya que favorece la formación de ciudadanos más conscientes, críticos y cuestionadores, poseedores de actitudes y valores que conducen a la mejora de la calidad de vida de la población, en un entorno sostenible y saludable. 
Cabe mencionar que los aportes formativos no se desarrollan a través del trabajo de un solo docente, sino a través de la participación de docentes de otras áreas del conocimiento, la participación de todos es fundamental, es en lo colectivo que los avances serán más significativos. De este modo, iremos alejando el fantasma de la fragmentación de la enseñanza que tanto dificulta el proceso de enseñanza-aprendizaje de la educación ambiental.

En este sentido, se justifica la declaración de la Política Nacional de Educación Ambiental (PNEA) sobre la necesidad de que la Educación Ambiental esté presente en todas las modalidades y niveles de la educación, ya sea de carácter formal o no formal (Brasil, 1999a). En consecuencia, los Parámetros Curriculares Nacionales (PCN), publicados en 1998, presentan la Educación Ambiental como un tema transversal.

En esta perspectiva, en el cuaderno específico, indicar cómo incorporar la educación ambiental en los programas escolares (Brasil, 1998). Las Directrices Curriculares Nacionales para la Educación Ambiental (DCNEA), del mismo modo, abogan por el enfoque de la Educación Ambiental de forma transversal y la preservación del equilibrio del medio ambiente, entendiendo la defensa de la calidad ambiental como un valor inseparable del ejercicio de la ciudadanía (Brasil, 2012).

En esta misma línea, la Base Curricular Nacional Común - BNCC defiende la necesidad de una sociedad sostenible (Brasil, 2018). Cabe destacar que, en consonancia con la Ley de Directrices y Bases de la Educación - LDB (Brasil, 1996), la Base Curricular Nacional Común es un documento normativo, cuyo principal objetivo es establecer un conjunto orgánico y progresivo de aprendizajes esenciales, indicando los conocimientos y habilidades que espera que todos los alumnos desarrollen a lo largo de la Educación Básica. Así, se pretende fortalecer el diálogo universidad/escuela a través de esta experiencia práctica de educación ambiental, una práctica basada en el medio ambiente como forma de vida referida al entorno cotidiano, la escuela, el hogar, el barrio, el lugar de trabajo entre otros, involucrando los aspectos naturales, sociales y culturales especialmente la intercesión entre ellos.

La educación ambiental tiene como principio rector el redescubrimiento por parte de los seres humanos de su propio entorno, desarrollando un sentido de pertenencia que permita una actuación más responsable. La universidad, lugar de promoción y fundamento de los debates socioambientales, contribuye a la formación de ciudadanos críticos y reflexivos, capaces de intervenir en las realidades locales, regionales y globales.

Por lo tanto, es importante fortalecer la asociación universidad/escuela para componer el proceso formativo de los graduados en cuanto a la implementación del concepto de educación para la sostenibilidad, que permite una discusión y reflexión más amplia sobre la cuestión socioambiental local/global, tratando el medio ambiente como una forma de vida. Se cree que es necesario ensayar nuevos caminos de formación, con el objetivo de contribuir a la formación de los profesores y a la construcción de currículos que tengan espacio para lo nuevo, para la construcción y resignificación del conocimiento, revisando las limitaciones de la formación tradicional.

\section{Metodología}

La investigación cualitativa se desarrolló a través de la base teórica relativa a la Educación Ambiental, la observación interactiva en la enseñanza de las Ciencias Naturales en la educación primaria con el fin de abordar el tema ambiental transversal en todas las asignaturas de la educación básica. Entre las características de este tipo de investigación, Pereira et al. (2018) destacan el hecho de tener como fuente directa de datos el entorno natural y como instrumento principal al investigador; los datos recogidos son predominantemente descriptivos y el investigador se preocupa por el significado que las personas dan a las cosas y a sus vidas. Así, el análisis de los datos constituye un proceso inductivo.

Durante el componente curricular de Práctica Docente: temas transversales, dictado en el $8^{\circ}$ período de la carrera de Ciencias Biológicas de la Facultad de Ciencia y Tecnología Profesor Dirson Maciel de Barros, se desarrollaron escritos reflexivos 
sobre las discusiones realizadas en el aula. Los temas transversales fueron elegidos por los estudiantes de grado de la disciplina y ejecutados a través de un proyecto de extensión. Así, para este estudio se seleccionó el material relativo a la escritura de las reflexiones sobre la conferencia: "Conciencia Ambiental" por la emergencia en el posicionamiento de sí mismos como futuros profesores de Ciencias y Biología frente a la crisis ambiental en la que estamos viviendo y la importancia de la comprensión de lo que las concepciones de la Educación Ambiental están constituyendo en su formación.

Teniendo en cuenta los conocimientos teóricos que orientan la Educación Ambiental se sugirió al profesor de Ciencias Naturales, un enfoque del tema transversal "Medio Ambiente", con la intención de provocar en los alumnos la pregunta sobre la Educación Ambiental en la escuela.

Tras el consentimiento de la propuesta se desarrollaron dos actividades en el aula en diferentes clases de $9^{\circ}$ grado de primaria de la Escuela Estatal Benigno Pessoa de Araújo. Cada clase estaba compuesta por unos 20 alumnos, por lo que existía la posibilidad de la composición de cuatro grupos. La primera actividad dirigida a los alumnos, requería que cada grupo, a través del grupo de estudio, uniera sus conocimientos sobre: "¿Qué es la educación ambiental?" A continuación, presenta "¿qué sugerencias y/o mecanismos podría utilizar el grupo para reducir el consumo de materiales como las bolsas de plástico, las botellas de PET y las latas en el medio ambiente?" Cada equipo tuvo que presentar a través de un debate sus entendimientos y propuestas según el enfoque del tema transversal.

La segunda actividad fue la inserción del juego "Recicla Mundo" para la verificación y reconstrucción de los conocimientos de los alumnos sobre la recogida selectiva y el reciclaje. La actividad de entretenimiento "Recycle World" es un juego de mesa con recorrido y movimiento a través de dados. El juego se compone de 01 tablero, 02 dados, 01 reloj de arena, 06 marcadores (verde/vidrio, amarillo/metal, rojo/plástico, azul/papel, negro/madera, marrón/orgánico), 80 tarjetas de preguntas (tarjeta de residuos), 96 fichas de residuos de plástico del mismo color que los marcadores. El juego permite la participación de 3 a 6 jugadores, mayores de doce años, y su duración media es de 40 minutos. El objetivo de cada participante es completar las ocho casillas del "campo de producción y consumo" y las ocho casillas del "campo de recogida y reciclaje".

La realización de esta investigación se llevó a cabo durante cuatro días, y tras la observación y participación en el abordaje del tema transversal "Medio Ambiente" en la enseñanza de las Ciencias Naturales, se examinó la investigación en cuanto a las expectativas y limitaciones presentadas en las actividades realizadas en las aulas.

\section{Referencia Teórica}

\subsection{La educación ambiental y su perspectiva en la enseñanza de las ciencias como tema transversal}

La inserción de la educación ambiental en el ámbito escolar influye en la sensibilización del alumno para la adopción de un nuevo comportamiento, proporcionando ayuda al alumno en su análisis crítico de la conducta humana, que ha llevado a la degradación sin medida de las consecuencias de los recursos naturales.

En este sentido, Luca, dos Santos, Del Pino y Pizzato (2018) entienden que la educación ambiental en la escuela, debe abordar una actividad continua; de carácter interdisciplinar; con un perfil multidimensional; centrada en la participación social y en la solución de los problemas ambientales; dirigida a cambiar valores, actitudes y comportamientos sociales. Para lograr este objetivo, es necesario que los profesores desarrollen en sus clases la educación ambiental con un enfoque crítico centrado en la sostenibilidad, en una perspectiva interdisciplinar, transversal y contextualizada.

En los cursos de pregrado, durante la formación docente, las disciplinas que involucran la temática ambiental tienen menús muy diferentes, no presentando muchas veces las pautas de trabajo interdisciplinario, transversal, crítico, reflexivo y contextualizado, que los Parámetros Curriculares Nacionales de Medio Ambiente recomiendan para la educación primaria y secundaria, siendo muchas veces insertados de manera optativa, llevando a los egresados a una formación heterogénea, y a veces 
limitada, en relación a la educación ambiental. (Leite \& Rodrigues, 2018). Como consecuencia, muchos profesores no se sienten cómodos para tratar temas medioambientales en sus clases, normalmente porque no dominan los conceptos inherentes al tema o no saben cómo relacionarlos con el contenido de las asignaturas que imparten.

Por lo tanto, teniendo en cuenta la importancia de la educación ambiental y su obligatoriedad en los planes de estudio, resulta interesante evaluar las dificultades a las que se enfrenta el profesorado en la inserción y desarrollo de los temas ambientales en las asignaturas de primaria y secundaria.

\subsection{Formación del profesorado en la enseñanza de las ciencias}

La formación del profesorado y la búsqueda del desarrollo docente se reconocen como promotores de una mejor práctica que se refleja en la educación en las más variadas formas de enseñanza por lo que deben ser ponderadas, pues las peculiaridades de la profesión y su formación nos reportan nuevas perspectivas en la educación, viendo el hoy y también los retos para las próximas décadas, considerando que las transformaciones por las que la sociedad contemporánea está sometida e influenciada, son muy rápidas al involucrar, principalmente, las tecnologías y las relaciones humanas.

El área de formación docente ha tenido un gran desarrollo en los últimos 50 años, aumentando su influencia y originando un campo de investigaciones y publicaciones relevantes (Nóvoa, 2017). Según de Oliveira y Tiyomi Obara (2018) y Gadelha, R. M. (2020), la formación inicial y continua de los profesores se han constituido como temas de investigaciones y discusiones en la educación para la ciencia, con enfoques en las políticas educativas, el papel del profesor en la formación de ciudadanos, apreciando la formación de los profesores en la interfaz de la relación con los conceptos, el contenido, los estudios y la sociedad, entre otros.

Los profesores son considerados sujetos importantes en el proceso de enseñanza y aprendizaje, dotados de un saber y un hacer, que están en constante ejercicio de selección de pensamientos, acciones y metodologías a disposición de la enseñanza, que comienza en la formación inicial y continúa a lo largo de su carrera profesional. De este modo, será posible estimular la autorreflexión sobre el proceso de enseñanza en los programas de formación de profesores.

Por ello, la importancia de problematizar el proceso de formación de los educadores y, dentro de él, el currículo en la formación de pregrado, en vista de una reconstrucción permanente del mismo, para atender las necesidades de la realidadentorno; esto implica reflexionar sobre los conflictos sociales, sobre la formación de los docentes, Bastos (2017, p.5 ) aborda que los profesores "[...] revelan la existencia de un saber pedagógico que no se adquiere ni se construye inmediatamente, sino que se va construyendo a lo largo de la carrera, es decir, a medida que impregna los métodos de enseñanza". En esta perspectiva, es necesario considerar la formación del profesorado como un proceso inicial y continuo que debe responder a los retos de la vida escolar cotidiana, la contemporaneidad y el avance tecnológico. El profesor es uno de los profesionales que más necesita actualizarse, compaginando la tarea de enseñar con la de estudiar. Transformar esta necesidad en un derecho es fundamental para el logro de su valorización profesional y el desempeño en niveles de competencia requeridos por su propia función social.

Según Gil-Pérez y Vilches (2017), los profesores en ejercicio o en formación deben romper las visiones simplistas de la enseñanza de las ciencias, reconociendo las carencias de su formación, para luego ir en busca del "saber" y del "saber hacer" en el desarrollo de una enseñanza de calidad. Las necesidades de formación del profesorado se basan en: conocer la materia a enseñar, conocer y cuestionar el pensamiento didáctico espontáneo, adquirir conocimientos teóricos sobre el aprendizaje y la enseñanza de las ciencias, que le permitan criticar la enseñanza tradicional y descontextualizada, repensar la preparación de las actividades y su realización, reflexionar sobre cómo evaluar el proceso de enseñanza y aprendizaje, adquirir la formación necesaria para asociar la enseñanza y la investigación. 
Teniendo en cuenta la complejidad de la formación del profesorado, que es un proceso largo que no termina con la obtención del título, es necesario buscar constantemente los conocimientos y las habilidades que no se adquirieron durante el tiempo de la formación inicial, así como las actualizaciones de la enseñanza que han surgido posteriormente. Ante esto, Bonzanini y Bastos (2009, p. 3) refuerzan la necesidad de que "[...] los profesores tengan posibilidades de formación y actualización permanente, diversa y de calidad". Por lo tanto, la formación del docente principiante presenta necesidades de transformaciones vinculadas a su formación y trayectoria (Jardilino; Sampaio, 2019). De este modo, será posible estimular la autorreflexión sobre la enseñanza en los programas de formación de profesores. Por ello, la importancia de problematizar el proceso de formación de los educadores y, dentro de él, el currículo en la formación de pregrado, en vistas a una reconstrucción permanente del mismo, para responder a las necesidades de la realidad-entorno; esto implica reflexionar sobre los conflictos sociales, culturales, políticos, económicos, tecnológicos y ambientales en los que se inserta la universidad hoy.

\subsection{La experiencia docente como base para la enseñanza del medio ambiente en la educación básica}

La perspectiva de la enseñanza sobre el tema de la Educación Ambiental (EE) y el medio ambiente se insertó en las escuelas a través de los Parámetros Curriculares Nacionales (PCN) que dieron apoyo y orientación a los profesores en el trabajo con los estudiantes y las posibles acciones a implementar. Y es en este documento donde surge el enfoque, transversal y pedagógico sobre el tema Medio Ambiente, para exponer la EE en todos los años y áreas de conocimiento de la educación primaria.

Con ello, surgen cada vez más movimientos desde la perspectiva no sólo de discutir los problemas e impactos ambientales y la relación del hombre con ellos, sino principalmente, de encontrar formas que puedan concienciar sobre el entorno en el que nos desenvolvemos.

Es importante destacar que, incluso después de la implementación de la Política Nacional de Educación Ambiental (LEY 9.795/99), que contiene los principios básicos de la Educación Ambiental en Brasil, y de la publicación de los Parámetros Curriculares Nacionales (Brasil, 1998a), que incluyen el medio ambiente como tema transversal, las concepciones y prácticas de los profesores en relación con las cuestiones ambientales no han cambiado significativamente.

Esta constatación permite afirmar que es urgente la formación de los docentes para trabajar con la temática ambiental, dando lugar a prácticas que puedan contribuir a la formación de un "sujeto ecológico", según la concepción de Moura Carvalho (2017), capaz de comprender su entorno natural, correlacionándolo con los aspectos sociales, políticos, económicos y culturales, y que permita ampliar los horizontes de actuación de los individuos, buscando la sostenibilidad del planeta.

Con la formación inicial se pretende que los profesores-discentes comprendan la relevancia de la organización de su trabajo en el espacio escolar desde la perspectiva de asegurar el aprendizaje y el desarrollo humano de los alumnos de la educación básica, tal como establece el Plan Nacional de Educación (Brasil, 2019). La lectura y la reflexión de las referencias teóricas proporcionadas en las disciplinas se destacan como puntos clave en esta formación, considerando el objetivo de profundizar las discusiones sobre la articulación entre el conocimiento de las Ciencias Naturales y las diversas áreas relacionadas, a partir de la realidad escolar.

La perspectiva de la integración de los conocimientos requiere que los profesores amplíen su comprensión de la totalidad de los componentes curriculares, tanto en forma de asignaturas como de otras posibilidades de organización del conocimiento escolar.

En este escenario, se propone una serie de actividades de reflexión sobre las herramientas de planificación en la organización del trabajo pedagógico. Las reflexiones individuales y colectivas integraron la oportunidad de conocer a fondo el 
espacio escolar con el reconocimiento de que las peculiaridades locales pueden influir en la actividad docente, favoreciendo o dificultando el aprendizaje.

\section{Resultados y Discusión}

A la hora de planificar el curso, pensamos que sería interesante insertar a los estudiantes de grado en las escuelas, ya que la experiencia en el entorno escolar sería fundamental para familiarizarlos con su futuro campo de actividad profesional.

Los alumnos formaron equipos y los temas se sortearon entre ellos. Dentro del tema central, podían elegir temas específicos y preparar el proyecto para trabajar en la educación básica. Así, cada grupo elaboró su proyecto, trazando metas y objetivos a alcanzar, además de cumplir con lo propuesto en el plan docente de la asignatura. Destacamos que cuando las actividades propuestas se aplicaron en la escuela, el profesor de la asignatura las acompañó durante el desarrollo del proyecto, para su posterior evaluación y aportes al aprendizaje de los alumnos de grado.

El primer ejercicio propuesto a los alumnos del $9^{\circ}$ grado de las escuelas primarias de la Escuela Estatal Benigno Pessoa de Araújo, pretendía buscar la comprensión de la definición de Educación Ambiental, y la presentación de los recursos utilizados por los alumnos para la reducción de materiales como botellas PET, bolsas de plástico y latas en el medio ambiente.

Al principio, se observó que los alumnos no parecían motivados para aprender ciencias naturales, permaneciendo en el aula sin participar ni dialogar con el profesor, ni siquiera con los demás presentes en el aula. Aunque el planteamiento se centra en la observación de un tema transversal, el profesor de la asignatura también se dio cuenta de que la estancia de alguien diferente al entorno del aula, ya estimulaba a los alumnos a querer participar en la dinámica propuesta. Las observaciones realizadas al aplicar el primer ejercicio de abordaje de la Educación Ambiental como tema transversal en la enseñanza de las Ciencias Naturales, permitieron identificar los eventuales conocimientos entre los cuatro grupos conformados.

A lo largo de las discusiones sobre la definición de la Educación Ambiental se pudo constatar que algunos estudiantes no tenían sus propios puntos de vista sobre el verdadero significado de este proceso. Algunos repitieron expresiones definidas teóricamente por los autores, pero no parecían conocer el significado o la importancia de la Educación Ambiental. Otros explicaron que la Educación Ambiental "es la práctica de no tirar basura en las calles, es ser cuidadosos con el lugar donde vivimos. Así, se denota una definición segmentada de la Educación Ambiental.

Además, se ha podido comprobar que pocos alumnos comprenden la relevancia de una conducta diferenciada frente a los problemas medioambientales. Esta pequeña porción expuso claramente la definición de Educación Ambiental y su importancia como procedimiento educativo transformador para el mantenimiento del medio ambiente, el bienestar social y promotor de la calidad de vida. En el primer ejercicio también se pudo comprobar la conciencia medioambiental de los alumnos, a partir de las propuestas sugeridas para la reducción de materiales como las botellas de PET, las bolsas de plástico y las latas en el medio ambiente.

A lo largo de esta actividad, la idea de introducir el grupo de estudio en la enseñanza de los estudiantes resultó eficaz para el aprendizaje de los adolescentes que aceptaron participar en la discusión. Entre las propuestas sugeridas por un grupo estaba la sensibilización de la comunidad en general a través de la educación. Esta educación, según el grupo, podría llevarse a cabo mediante la distribución de folletos en supermercados e instituciones educativas del municipio. Los folletos contendrían directrices sobre cómo manejar adecuadamente cada tipo de basura, en cuanto a su almacenamiento y correcta eliminación.

Al ser preguntados por el educador sobre la fragilidad de la estrategia propuesta que podría favorecer el aumento de la cantidad de residuos de papel en las calles, los alumnos afirmaron que "de hecho la cantidad de basura podría crecer, pero si al menos un individuo leyera el folleto y no lo tirara a la calle la estrategia no sería en absoluto inadecuada". Otro grupo señaló como solución el cambio de las bolsas de plástico por el uso de cajas de cartón y bolsas ecológicas en los supermercados para 
reducir la cantidad de materiales en el medio ambiente. Según este grupo, la dirección municipal sería capaz de proponer un cambio en la conducta de los supermercados, así como recomendar a la población que separe su basura para realizar una correcta recogida selectiva. Sin embargo, si la población y los supermercados no ajustaran su estrategia se verían sometidos al pago de multas, ya que las cuestiones económicas son más importantes que la Educación Ambiental.

El tercer grupo recomendó reciclar materiales para reducir la cantidad de residuos en el medio ambiente. Según los estudiantes "el reciclaje ya aboga por la recogida selectiva de materiales, este hecho por sí solo ya permitiría a la población urbana concienciarse de la necesidad de separar adecuadamente los residuos, y no tirarlos a la calle, a las cunetas o al río". Este grupo también afirmó que "es la municipalidad la que tiene que tener el reciclaje como una forma ambientalmente viable para la población, porque la comunidad está dispuesta a reducir la cantidad de basura, pero si la municipalidad no muestra opciones para la eliminación de la basura, ésta seguirá siendo enviada al vertedero y, en consecuencia, al medio ambiente".

Al final de estas observaciones, el profesor informó que la perspectiva del tema Educación Ambiental es pequeña en el aula. Por lo general, su enfoque se produce a través de minicursos impartidos por universidades en colaboración con la escuela. Sin embargo, no se redujo a esta controversia haciendo mención al artículo 2 de los Lineamientos Curriculares Nacionales para la Educación Ambiental (2012), estando así de acuerdo en que "la Educación Ambiental es una extensión de la educación, siendo un compromiso que tiene la intención de la práctica social, la cual debe dar al desarrollo particular una característica social en su relación con la naturaleza y con los demás seres humanos, pretendiendo intensificar este desempeño del hombre para hacerlo lleno de práctica social y ética ambiental".

El segundo ejercicio dirigido al procedimiento alternativo de Educación Ambiental en la enseñanza de las ciencias naturales fue la introducción de la actividad de entretenimiento "Recicla mundo". El juego didáctico fue calificado como fácil, de excelente manejo, y fue bien recibido por los alumnos de las clases de $9^{\circ}$ grado. Se observó que durante la realización de la actividad recreativa hubo interacción entre los alumnos. También se percibió la indispensabilidad de conocimientos previos sobre los temas relacionados con la Educación Ambiental. Este hecho permite entender que la Educación Ambiental no debe ser abordada como un tema independiente de la formación de los alumnos, ya que el tema transversal "Medio Ambiente" es capaz de aportar nuevos comportamientos ante las nuevas expectativas ambientales y los debates de carácter político y social en los que está inmersa toda la sociedad.

Otra consideración relativa a la aplicabilidad del juego didáctico está asociada a la planificación sobre cómo debe realizarse una actividad lúdica. Lo lúdico no debe sustituir el enfoque de los temas o contenidos abordados por el profesor. El propósito de la actividad lúdica debe ser añadir el contenido y los temas presentados por el educador a su inserción como práctica en el aula. Ya que el alumno es un aprendiz y reconstruye sus conocimientos según la metodología utilizada por el profesor.

Durante el transcurso del juego "El mundo del reciclaje", los alumnos tuvieron la oportunidad de entender las respuestas correctas a las preguntas del juego. Los alumnos estuvieron atentos a las normas establecidas y estimularon su participación. La actividad lúdica también proporcionó una oportunidad para construir el conocimiento y la participación activa del alumno. Sin embargo, algunos estudiantes tuvieron dificultades con las preguntas formuladas a lo largo del juego.

Se comprobó que la mayoría de los alumnos sabía distinguir correctamente los términos Reducir, Reutilizar y Reciclar. Sin embargo, estos alumnos no tenían los conocimientos reales sobre la recogida selectiva. Otros alumnos demostraron que reconocían los principales colores de los cubos de basura de la escuela, como el verde, el amarillo, el rojo y el azul. Pero estos mismos alumnos no sabían qué tipo de residuos debían indicarse a cada recolector según su color.

A medida que se desarrollaba el juego, se pudo constatar la falta de conocimientos sobre el tiempo de descomposición de los materiales y sobre las formas alternativas que se pueden encontrar para tratar los residuos sólidos. A partir de los resultados 
obtenidos, se entendió que la inserción de juegos didácticos para el abordaje de temas transversales en la enseñanza de las Ciencias Naturales es una posibilidad viable.

El examen de este enfoque diferenciado permitió comprender que el uso de la actividad lúdica es capaz de provocar la motivación de los alumnos despertando su interés por querer adquirir conocimientos a través de los contenidos y posibles temas de discusión propuestos en el aula. Por lo tanto, la perspectiva de la Educación Ambiental como tema transversal y la propuesta diferenciada facilitaron que el alumno se apropiara de nueva información y reconstruyera sus conocimientos a partir del relevamiento de temas relacionados con el medio ambiente.

Se destaca que el proyecto organizado y puesto en práctica en la disciplina de Práctica Docente V fue un momento muy productivo, de diversos aprendizajes e intercambios entre los participantes, la Universidad y la escuela. Se supone que ha contribuido a la autonomía de los graduados, como futuros educadores, y a que piensen en sus prácticas pedagógicas en la búsqueda de la transformación del proceso de enseñanza y aprendizaje.

\section{Consideraciones Finales}

El desarrollo de esta investigación proporcionó una ocasión propicia para introducir en la enseñanza de las Ciencias Naturales un posible enfoque del tema transversal "Medio Ambiente". Teniendo en cuenta la propuesta presentada a los alumnos, éstos pudieron reconocer el entorno del que forman parte y reconstruir su visión sobre el verdadero significado de la Educación Ambiental.

La legislación define las formas de trabajo de la educación ambiental en todos los niveles de la enseñanza, y se puede observar la importancia que dan los profesores a la presencia de los temas ambientales en la educación escolar.

La inserción del juego didáctico fue eficaz para abordar el tema transversal, ya que permitió a los alumnos promover ideas que orientan las acciones para cambiar por un mundo mejor. En cuanto al enfoque de la educación ambiental en la enseñanza de las ciencias, se constataron las expectativas y las limitaciones experimentadas a través de una observación participativa en diferentes cursos del $9^{\circ}$ grado de las escuelas primarias. Así, se cree que el enfoque diversificado de temas transversales como la Educación Ambiental se suma al aprendizaje de los alumnos para estimularlos a asumir una conducta consciente respecto a las cuestiones socioambientales.

Se destacó entre los participantes el interés y la importancia de un material de apoyo que contenga recursos didácticos, legislación y cuestiones medioambientales, considerándose una valiosa ayuda en la preparación de las clases, lo que refuerza el vacío existente en la formación del profesorado. Se hace necesario evaluar y definir formas de minimizar y revertir esta carencia en la formación del profesorado, con el objetivo de abordar de forma efectiva una educación ambiental crítica en la educación primaria y secundaria.

Desde el punto de vista de la formación, creemos en la capacitación de un profesor en la formación inicial como punto de partida para la constitución de un profesor cualificado. Por lo tanto, estudiar y reflexionar sobre el sesgo transversal en el que la EA necesita ser trabajada en la educación básica es esencial para cumplir con las Leyes instituidas sobre la EA, permitiendo a los estudiantes ver la interrelación de la EA en todos los componentes del currículo, en consecuencia de la vida en el planeta.

De ahí que entendamos como necesario un plan que implique más discusiones y debates centrados en el tema de la educación ambiental donde se contemplen todos los sujetos, es decir, además de los alumnos, los profesores.

Por último, se considera que los resultados de la investigación ponen de manifiesto que la Educación Ambiental debe ser discutida tanto en la formación inicial como en la formación continua de los profesores para buscar el desarrollo de un concepto que esté de acuerdo con la legislación vigente para que, entonces, los profesores puedan proporcionar a los alumnos de la Educación Básica una educación ambiental de calidad. 
La Universidad juega un papel importante en la sociedad a la hora de formar a los futuros profesionales, dentro de este contexto, con el resultado de la experiencia de la asociación Universidad/Escuela, es necesario implementar en los planes de estudio de los cursos de pregrado instrumentos para una formación no sólo científica, sino social ciudadana haciendo surgir individuos críticos, cuestionadores de la realidad local y global. La práctica de una educación ambiental contextualizada es esencial para que los futuros profesores construyan conocimiento, identificando el problema, buscando colectivamente alternativas aplicables a la realidad del alumno.

Se entiende que el desarrollo de prácticas educativas a partir de los contenidos de las ciencias naturales de relevancia social y ambiental se presenta como una alternativa esencial e interesante para la formación integral de los estudiantes para el ejercicio de la ciudadanía. Sin embargo, es necesaria una reformulación en los cursos de pregrado en la formación inicial de los profesores, además de la inversión en la formación continua de manera significativa, centrada en la inserción de las cuestiones ambientales en la práctica. Como sugerencias para el trabajo futuro, esta investigación sirve para incrementar la educación continua para que poco a poco el tema ambiental, tan importante para toda la humanidad, sea parte de las escuelas de educación básica.

Así, se destaca la necesidad de una formación más sólida del profesorado para la actividad docente en Educación Ambiental, ya sea en la formación inicial, ya sea en la continua. También se destaca la posibilidad de realizar cursos de formación inicial en Educación Ambiental que proporcionen espacios de fundamentación teórica y de discusión y puesta en común de experiencias prácticas de Educación Ambiental.

\section{Referencias}

Brasil. (1996). Ley $\mathrm{n}^{\mathrm{o}}$ 9.394, de 20 de diciembre de 1996. Establece las directrices y las bases de la educación nacional. <https://www.planalto.gov.br/ccivil_03/Leis/L9394.htm> Consultado el: 17 jul. 2021.

Brasil. (1997). Ministerio de Educación. Parámetros del plan de estudios nacional. Brasilia, DF: MEC/SEF.

Brasil. (1998). Parámetros Curriculares Nacionales: tercer y cuarto ciclo de la Educación Básica: temas transversales. Brasilia: MEC/SEF. 436.

Brasil. (1999). Ley n 9.795, de 27 de abril de 1999. Discurso sobre la educación Educación Ambiental, por el que se establece la Política de Educación Ambiental y otras disposiciones. <http://www.lei.adv.br/9795-99.htm>.

Brasil. (2013). Directrices del plan de estudios nacional para la educación ambiental. Directrices generales del plan de estudios nacional para la educación básica, $515-542$.

Brasil. (2017) Ministerio de Educación. Consejo Nacional de Educación. Consejo en pleno. Ley No 13.415/2017. Modifica las Leyes nº 9.394, del 20 de diciembre de 1996, que establece las directrices y bases de la educación nacional, y 11.494, del 20 de junio de 2007, que regula el Fondo de Mantenimiento y Desarrollo de la Educación Básica y la Valoración de los Profesionales de la Educación, la Consolidación de las Leyes del Trabajo - CLT, aprobada por el Decreto-Ley n ${ }^{\circ}$ 5. 452, del 1 de mayo de 1943, y el Decreto-Ley 236, del 28 de febrero de 1967; deroga la Ley 11.161, del 5 de agosto de 2005; e instituye la Política de Fomento de la Implantación de Escuelas Secundarias de Tiempo Completo.

Brasil. (2018). Ministerio de Educación. Consejo Nacional de Educación. Conselho Pleno. Resolución n 4 , de 17 de diciembre de 2018 . Institui a Base Nacional Comum Curricular na Etapa do Ensino Médio (BNCC-EM), como etapa final da Educação Básica, nos termos do artigo 35 da LDB, completando o conjunto constituído pela BNCC da Educação Infantil e do Ensino Fundamental, com base na Resolução CNE/CP n 2/2017, fundamentada no Parecer CNE/CP $n^{\circ} 15 / 2017$

Brasil. (2019). Ministerio de Educación - MEC. Base Nacional Comum Curricular. Brasilia, $2019 . \quad$ Disponible em: http://basenacionalcomum.mec.gov.br/images/BNCC_EI_EF_110518_versaofinal_site.pdf Consultado el 8 de agosto de 2021.

Bastos, M. J. A (2017). Formación de profesores para la educación básica. Revista Científica Multidisciplinar Núcleo del Conocimiento, $14(2)$, 82-97.

Bonzanini, T. K.; Bastos, F. (2009). Formación continua para profesores de ciencias: algunas reflexiones. Anales. VII Encuentro Nacional de Investigación en Enseñanza de las Ciencias.

Luca, A. G., dos Santos, S. A., Del Pino, J. C., \& Pizzato, M. C. (2018). Experimentación contextualizada e interdisciplinar: una propuesta para la enseñanza de las ciencias. Revista Insignare Scientia-RIS, 1(2).

Gadelha, R. M. (2020). La formación inicial y continua del profesorado. Anais VII CONEDU-Ediç o Online... Campina Grande: Realize Editora.

Gil Pérez, D., y Vilches, A. (2017). Educación para la sostenibilidad y educación en derechos humanos: dos campos que deben vincularse. Educación para la Sostenibilidad y Educación en Derechos Humanos: dos campos que deben vincularse, 79-100. 
Research, Society and Development, v. 10, n. 12, e51101220166, 2021

(CC BY 4.0) | ISSN 2525-3409 | DOI: http://dx.doi.org/10.33448/rsd-v10i12.20166

Gomes, K. R. F. P., do Nascimento, S. M., Leite, E. F., \& Alves, J. L. (2020). Sostenibilidad y Políticas Públicas: Tratamiento en Educación Ambiental Contemporánea. Research, Society and Development, 9(9), e707997880-e707997880.

Gomes, K. R. F. P., Nascimento, S. M. d., Rosa, R. V., Silva, A. B. d., Leite, E. F., Araújo, M. A. V., . . Rosas, M. d. L. Á. C. (2020). La gestión sostenible desde la perspectiva de las prácticas organizativas en diferentes sectores. Research, Society and Development, 9(11). e2199119706-e2199119706.

Gomes, K. R. F. P., do Nascimento, S. M., \& Alves, J. L. (2020). La Sostenibilidad en la Enseñanza Superior: un estudio de caso de la relación profesorestudiante en el curso de Administración de la FCAP/UPE. Research, Society and Development, 9(12), e19791210997-e19791210997.

Jardilino, J. R. L., \& Sampaio, A. M. M. (2019). Desarrollo profesional del profesorado: reflexiones sobre las políticas públicas de formación del profesorado. Educación y formación, 4(10), 180-194.

Layrargues, P. P. (2018). El servilismo al capital: la educación ambiental bajo el signo del antiecologismo. Research in Environmental Education, 13(1), 28-47.

Leite, R. F., \& Rodrigues, M. A. (2018). Aspectos socio-científicos y la cuestión medioambiental: una dimensión de la alfabetización científica en la formación de profesores de química. Journal of science and mathematics teaching, 9(3), 38-53.

Moura Carvalho, I. C. (2017). La educación ambiental: la formación del sujeto ecológico. Cortez Editora.

Pereira, A. S., Shitsuka, D. M., Parreira, F. J., \& Shitsuka, R. (2018). Metodologia da pesquisa científica.

Oliveira, A. L., \& Tiyomi Obara, A. (2018). La enseñanza de las ciencias por indagación: experiencias y prácticas reflexivas de los profesores en formación inicial y continua. Investigaciones sobre la enseñanza de las ciencias, 23(2).

Santos Pereira, M. C., Farias, L. A., \& Neiman, Z. (2020). Educação Ambiental e política pública educacional: uma abordagem interdisciplinar na perspectiva da intervenção social a partir do projeto Ocupe a Praça (São Paulo, SP). Revista Brasileira De Educação Ambiental (RevBEA), 15(1), $209-244$.

Sauvé, L. (2017). Educación para la eco-ciudadanía. Diccionario crítico de los temas y conceptos de la Educación en. París: L'Harmattan , 56-65.

Reigota, M. (2017). Qué es la educación ambiental. Brasiliense. 